\title{
Review
}

\section{Investigating Acupuncture Using Brain Imaging Techniques: The Current State of Play}

\author{
George T. Lewith ${ }^{1}$, Peter J. White ${ }^{2}$ and Jeremie Pariente ${ }^{3}$ \\ ${ }^{1}$ Primary Medical Care, School of Medicine, University of Southampton, ${ }^{2}$ School of Health Professions and \\ Rehabilitation Sciences, University of Southampton, UK and ${ }^{3}$ Neurology Department, Purpan Hospital, \\ Toulouse, France
}

\begin{abstract}
We have systematically researched and reviewed the literature looking at the effect of acupuncture on brain activation as measured by functional magnetic resonance imaging and positron emission tomography. These studies show that specific and largely predictable areas of brain activation and deactivation occur when considering the traditional Chinese functions attributable to certain specific acupuncture points. For example, points associated with hearing and vision stimulates the visual and auditory cerebral areas respectively. Pain, however, is a complex matrix that is intimately intertwined with expectation. Acupuncture clearly affects this matrix in both specific and non-specific manner that is consistent with its specific clinical effects, as well as the effects of expectation on pain relief. This article summarizes the current imaging literature.
\end{abstract}

Keywords: acupuncture - imaging - review

\section{Introduction}

Interest in investigating acupuncture with various imaging techniques have been growing since the mid 1990s. The development of imaging techniques, such as positron emission tomography (PET) and functional magnetic resonance imaging (fMRI), have opened a 'window' into the brain that allows us to gain an appreciation of the anatomy and physiological function involved during acupuncture in humans and animals non-invasively. Researchers such as Alavi (1) and Cho (2) were among the first to publish in this area. Their initial observations have subsequently been enhanced and further developed in relation to point specificity, effects in hearing, nausea and more generalized analgesic effects in both animal models and human experimental pain.

This review is not systematic but it is a comprehensive narrative review of the available literature with respect to imaging and acupuncture with the aim of defining both the current state of play and a future research strategy. One of the major problems within the field of acupuncture and

For reprints and all correspondence: Dr George Lewith, MA DM FRCP MRCGP, Complementary Medicine Research Unit, Primary Medical Care, Aldermoor Health Centre, Aldermoor Close, Southampton SO16 5ST, UK Tel: +44 (0)2380 241073; Fax: +44 (0)2380 701125; E-mail: gl3@ soton.ac.uk neuroimaging is the necessity for very careful and thoughtful trial design so that the researcher only asks one question at a time. With these two techniques (PET and fMRI), brain images are acquired while a subject/patient is performing a task. Brain images of each individual are then transformed to brain activation maps' using statistical software such as Statistical Parametric Mapping (3). Maps of individuals across the group are then collated and compared to another group or another task in the same group. Maps of 'difference of activation' are thus created enabling researchers to evaluate difference between tasks in a group of subjects or differences in activation between groups performing the same task. Differences of activation may be produced by both relative activation and deactivation of specific areas of the brain.

The advent of fMRI and PET allows us to see brain activation in a variety of clinical conditions, but it is often difficult to interpret these images and relate them to specific neurology when the non-specific effects of the treatment process also seem to influence brain activation and may be of great clinical relevance. While there has been some correlation with clinical outcome in experimental pain, there are no publications that to date have correlated clinical outcome in pathological conditions (such as osteoarthritis) with definitively induced acupuncture changes in brain activation. However, imaging

(C) The Author (2005). Published by Oxford University Press. All rights reserved.

The online version of this article has been published under an open access model. Users are entitled to use, reproduce, disseminate, or display the open access version of this article for non-commercial purposes provided that: the original authorship is properly and fully attributed; the Journal and Oxford University Press are attributed as the original place of publication with the correct citation details given; if an article is subsequently reproduced or disseminated not in its entirety but only in part or as a derivative work this must be clearly indicated. For commercial re-use, please contact journals.permissions@oupjournals.org 
offers us the potential for a far greater understanding of the neurophysiology of acupuncture, but its interpretation and clinical relevance remains problematic. Furthermore, experiments with imaging must be firmly grounded in a very thorough understanding of the sensations elicited by acupuncture as well as the neurophysiology and biochemistry that underpins the treatment. They must also be conducted in conjunction with clinicians who understand the modality and in association with clear clinical outcome criteria. This work was in its infancy when it was previously reviewed by Joannie Shen (4) some 5 years ago and our review summarizes and incorporates recent important advances.

\section{Identification of the Literature}

The papers, and therefore the literature, reviewed in this article have been derived from a combination of systematic searching within the common databases (EMBASE, PubMed, AMED, CINAHL, MEDLINE and CISCOM), as well as the use of personal contacts within the field simply to make sure that we have not omitted any major publication. However, we are only too aware that this is a rapidly changing field, and while we believe that this paper summarizes our current knowledge of brain imaging within acupuncture and draws out some of the important principles, we may not have been able to identify every single publication in this area.

\section{Traditional Chinese Medicine Revalidated: The Effect of Specific Acupuncture Points}

Cho et al.'s (2) work was the first to identify that specific areas of the visual cortex appeared to be activated in response to acupuncture points in the foot in the same way as it would respond to a stimulation from a light source shone into the eyes. The main point used to stimulate visual cortex was UB67; Cho and co-workers claimed that a difference in character of the subjects (i.e. Yin or Yan) accounted for variations in the pattern of response. This was point-specific and so could not be reduplicated through similar stimulation in a non-acupuncture point. A number of other visually active acupuncture points were manipulated and produced a similarly predictable response in accordance with traditional Chinese principles. The stimulation of auditory-related points similarly produced activation of the auditory cortex (5). Although the studies only involved small numbers of volunteers, the science appeared convincing. Further reduplication by others $(6,7)$ and also continuing work by Cho and co-workers seems to support this hypothesis. Siedentopf et al. (8) suggests similar phenomena to needle puncture may be elicited by soft laser acupuncture, a finding supported by Litscher et al. (9). However, Gareus et al. (10) throws some doubt on Cho's findings. Therefore, while it appears that it is probable that acupuncture-like stimulation of UB67 triggers specific activation of the occipital cortex, it is by no means a uniform and consistent finding.

Li et al. (11) looked at the possibility that a similar phenomenon may be elicited by using points specific to language in 17 healthy Mandarin speaking Chinese volunteers. They suggest that there is stronger activation in the left hemisphere and that electrical stimulation of two acupuncture points (implicated in languages, i.e. SJ8 and Du15) produced significant activation in an appropriate area of the brain, the right inferior frontal gyrus and in the left and right superior temporal gyrie. However, activation was not seen in the left inferior frontal gyrus and it is also clear that not all acupuncturists would agree that these points are related to language. Non-acupuncture points did not produce significant brain activation, so these researchers concluded that specific acupuncture points may have language-specific effects. Yoo et al. (12) noted that PC6 produces a well documented clinical response to acupuncture in nausea. They were able to demonstrate consistently that acupuncture-specific neural substrates are selectively activated in the left superior frontal gyrus, the anterior cingulate gyrus and the dorsomedial nucleus of the thalamus as well as nausea-specific substrates in the cerebellum. This did not occur with penetrating sham needling at a non-acupuncture point, thus suggesting a PC6-specific effect that potentially correlates with proven clinical effectiveness. Yan et al. (13) have demonstrated point-specific patterns using fMRI in 37 healthy volunteers while needling LI4 and Liv3 as compared to sham points. Common activation areas for LI4 and Liv3 were in the middle temporal gyrus and cerebellum, along with deactivation areas in the middle frontal gyrus and inferior parietal lobule when compared with sham points. Acupuncture at Liv3 evoked specific activation in the post-central gyrus, posterior cingulated, parahippocampal gyrus, BA 7, 19 and 41 , but deactivation at the inferior frontal gyrus, anterior cingulated, BA 17 and 18 when compared with sham. LI4 evoked specific activation in the temporal lobe but deactivation in the precentral gyrus, superior temporal gyrus, pulvinar and BA 8, 9 and 45 when compared with sham. They suggest that these different patterns may be related to specific therapeutic effects, although it is difficult to justify this based on data obtained from healthy volunteers receiving one acupuncture treatment.

\section{Pain Processing}

A variety of imaging studies have been published that look at the whole mechanism behind pain processing. A number of cortical areas have been shown to be involved in pain processing, these include the primary somatosensory cortex, the secondary somatosensory cortex, the insula, the anterior cingulate and the prefrontal cortex (14) as well as the hypothalamus and periaqueductal gray (PAG); the pain matrix. Derbyshire et al. (15) looked at 12 healthy volunteers using PET and subjected them to experimental pain with a $\mathrm{CO}_{2}$ laser. The pain was classified as: 'just painful', 'mildly painful' and 'moderately painful'. Again a wide range of active regions were shown to be responsive to pain, including the prefrontal inferior parietal and premotor cortices as well as the thalamus insula, primary somatosensory cortex and the ipsilateral perigenual cingulate cortex (15). Tracey et al. (16) have 
clearly defined the distraction techniques that may modulate clinical pain and activate the PAG. Petrovic et al. (17) have demonstrated that placebo analgesia involves the rostral anterior cingulate cortex and brainstem, again suggesting (through the use of PET) that both opiate and placebo analgesia are associated with increased activity in these endorphin-rich regions. Wager et al. (18) have found that placebo analgesia is related to decreased brain activity in pain-sensitive regions and that the effect of placebo analgesia is associated with expectation. The regions involved are the thalamus, insula and anterior cingulate cortex. These demonstrated increased activity during the anticipation of pain and diminished activity in the expectation of pain relief delivered by placebo. Fuente-Fernandez et al. (19) have also demonstrated that dopamine release in the nucleus accumbens is related to the expectation of reward, this may be involved in the placebo response to pain.

\section{The Modulation of Pain with Acupuncture}

Studies in this area have been conducted in both animal and human environments with experimental pain in healthy subjects. Chiu et al. (20) used three acupuncture points in rats to induce analgesia. They noted increased brain activity in the hypothalamus which was dependent on the extent of the stimulation. Liu et al. (21) also noticed a dramatically increased acupuncture-induced activation of the PAG in humans with both $\mathrm{Li} 4$ and a non-acupuncture point. It appeared PAG activation, while present as a consequence of non-acupuncture point stimulation, was substantially enhanced by stimulation of a true acupuncture point. Wu et al. (22) argues that acupuncture designed to provide analgesia involves the pain-related neuromatrix; this investigative group employed fMRI to evaluate the effects of electroacupuncture, mock electroacupuncture, minimal acupuncture and sham electroacupuncture on either real acupuncture points or nonacupuncture points in human healthy volunteers. Stimulation of real acupuncture points with electroacupuncture elicited significant activation over the hypothalamus, primary somatosensory motor cortex and rostral anterior cingulate cortex. The superior temporal gyrus and medial occipital cortex seemed to respond to minimal electroacupuncture, sham electroacupuncture and real electroacupuncture. Wu et al.'s group concluded that the hypothalamus and limbic system modulated the effects of electroacupuncture as an analgesic in a fairly non-specific manner. A similar conclusion was arrived at by Zang et al. (23), Wu et al. (24), Hui et al. (25), Kong et al. (26) and Hsieh et al. (27).

Fang et al. (28) looked at the method of needle stimulation to see if different areas were activated or de-activated in response to the nature of the stimulation. While they demonstrated similar areas of general activation in healthy subjects using Liv3 and G40, they were unable to demonstrate that specific needle manipulation techniques modified the response in the appropriate neuronal substrates. Biella et al. (29) also showed similar areas of general activation from acupuncture, which correlate closely with a wider pain matrix that have already been identified as modulating and managing expectation, placebo and pain. These papers and their main findings are summarized in Table 1.

It appears that there are substantial overlaps between the pain matrix that have already been identified within the brain and its response to placebo acupuncture and pain. There is also considerable overlap between the areas of placebo response and expectation and acupuncture, although a recent study of brain activation with PET involving patients in pain, by Pariente et al. (30), have clearly identified different areas of activation in response to touch with a needle, the expectation of acupuncture and real acupuncture, suggesting that real acupuncture not only activates areas associated with expectation and placebo response but also the ipsilateral insula that may represent a specific response to acupuncture in patients presenting with osteoarthritis pain. However, it does appear that there is a relatively 'plastic' pain matrix within the brain that is equally responsive to placebo and acupuncture with many overlapping areas. This might correlate quite closely with the large non-specific effects observed from many analgesic treatments, including acupuncture, in a clinical context $(31,32)$.

\section{Discussion}

Lewith and Vincent (33) have previously suggested that it may be appropriate to consider different underlying mechanisms behind the observed effect of acupuncture in different types of conditions. The preliminary data from neuroimaging would tend to support this observation in that specific neural substrates may be activated by the use of specific acupuncture points associated with widely acceptable traditional Chinese medical, non-painful, indications. However, in pain a far less specific response emerges containing overlapping neural substrates activated by both placebo and expectation.

These studies generally involve small numbers of subjects because of economic and practical situations and also raise a number of quite different specific issues. Should we be considering studies using imaging with respect to non-painful conditions in a different context to those looking at imaging in either experimental or clinical pain? The vast majority of studies on imaging and pain have been conducted in experimental pain, can we legitimately generalize from experimental pain into the clinical arena of chronic benign painful conditions? Imaging may allow us to differentiate between expectation, placebo and real acupuncture as Pariente et al. (30) suggest. However, considerable further investigation will be needed if we are to be anything more than tentative about these suggestions. Both technology and our understanding of neuroimaging are very much 'in development'. We must accept that this is a rapidly evolving field which is difficult to interpret with clarity.

It is quite possible that specific acupuncture points for non-painful conditions, such as PC6 in the treatment of nausea, may activate or inhibit very specific neuronal substrates. This 
Table 1. Summary of imaging studies, their subjects and conclusions (in date order of publication)

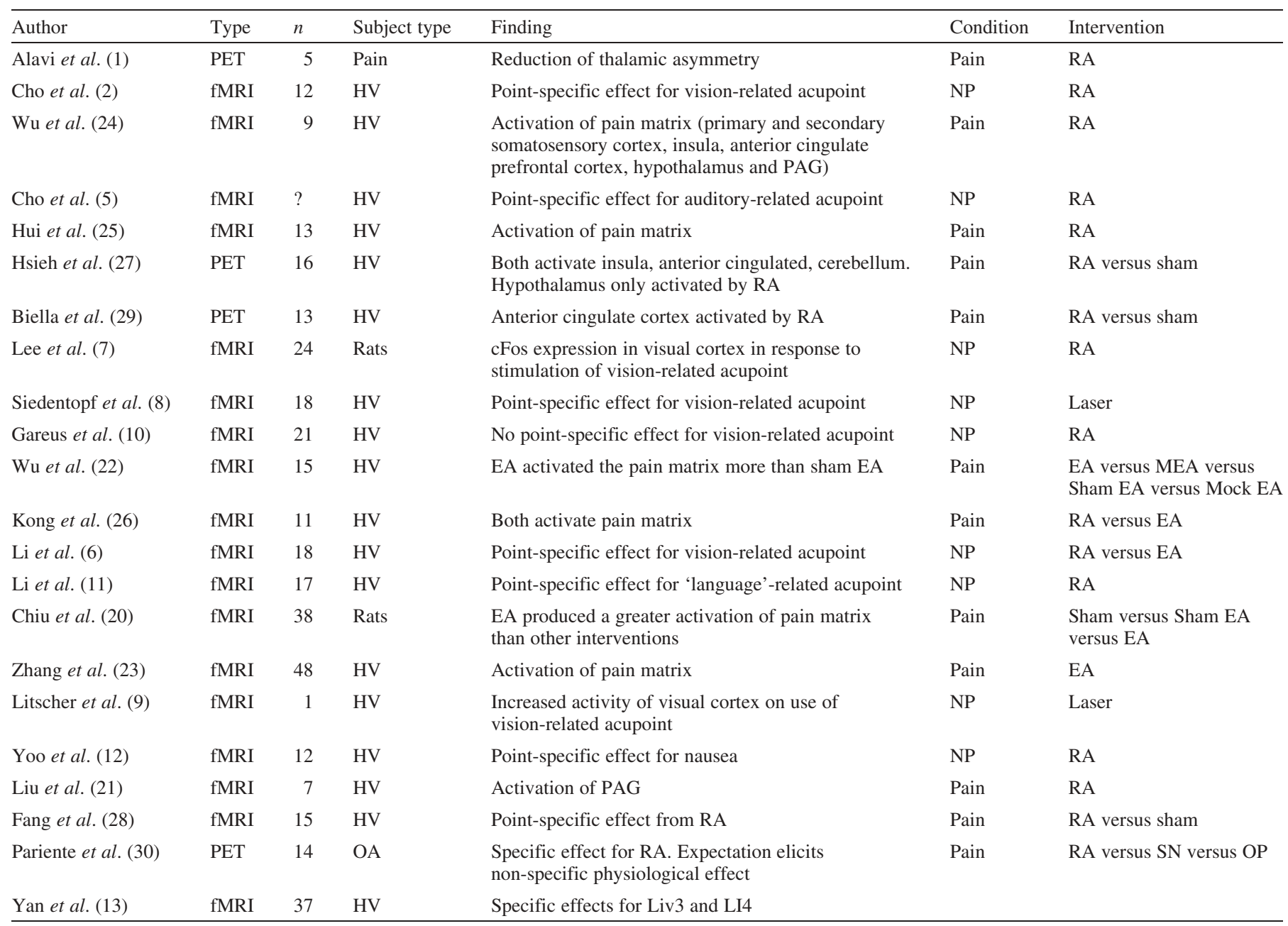

HV, healthy volunteer; OA, patient with Osteoarthritis; NP, non-pain; RA, real acupuncture; Sham, needling at a non-acupoint; EA, electroacupuncture; MEA, minimal (penetration) electroacupuncture; SN, Streitberger needle; OP, overt placebo.

may support, as Cho suggests, the assumptions inherent within traditional Chinese medicine. The effects of acupuncture in pain and possibly also in addiction may be far less point-specific as has been previously suggested from the available clinical evidence (33). To date, the limited work available from brain imaging would suggest that this might provide a reasonable series of hypotheses through which to investigate the effects of acupuncture using fMRI and PET.

\section{References}

1. Alavi A, LaRiccia P, Sadek A, Newberg A, Lee L, Reich H, et al. Neuroimaging of acupuncture in patients with chronic pain. J Altern Complement Med 1997;3:S41-53.

2. Cho Z, Chung S, Jones J, Park J, Park H, Lee H, et al. New findings of the correlation between acupoints and corresponding brain cortices using functional MRI. Proc Natl Acad Sci USA 1998;95:2670-3.

3. Frackowiak RSJ, Friston KJ, Frith CD, Dolan RJ, Price CJ, Zeki S, Ashburner J, Penny W. Human Brain Function. 2nd edition, Elsevier, 2004.

4. Shen J. Research on the neurophysiological mechanisms of acupuncture: review of selected studies and methodological issues. $J$ Altern Complement Med 2001;5:S121-7.
5. Cho Z, Na C, Wong E, Lee S, Hong I. Investigation of acupuncture using brain fMRI. In: Litscher G, Cho Z (eds). Computer Controlled Acupuncture. Berlin: Pabst Science Publishers, 2000, 45-64.

6. Li G, Cheung RTF, Ma Q-Y, Yang ES. Visual cortical activations on fMRI upon stimulation of the vision-implicated acupoints. Brain Imaging 2003;14:669-73.

7. Lee H, Park H-J, Kim SA, Lee HJ, Kim MJ, Kim C-J, et al. Acupuncture stimulation of the vision-related acupoint (Bl-67) increases c-Fos expression in the visual cortex of binocularly deprived rat pups. Am J Chin Med 2002;30:379-85.

8. Siedentopf C, Golaszewski SM, Mottaghy FM, Ruff CC, Felber S, Schlager A. Functional magnetic resonance imaging detects activation of the visual association cortex during laser acupuncture of the foot in humans. Neurosci Lett 2002;327:53-6.

9. Litscher G, Rachbauer D, Ropele S, Wang L, Achikora D, Fazekas F, et al. Acupuncture using laser needles modulates brain function: first evidence from functional transcranial Doppler sonography and functional magnetic resonance imaging. Lasers Med Sci 2004;19: 6-11.

10. Gareus I, Lacour M, Schulte A, Hennig J. Is there a BOLD response of the visual cortex on stimulation of the vision-related acupoint GB 37?. J Magn Reson Imaging 2002;15:227-32.

11. Li G, Liu H-L, Cheung RTF, Hung Y-C, Wong KKK, Shen GGX, et al. An fMRI study comparing brain activation between word generation and electrical stimulation of language-implicated acupoints. Hum Brain Mapp 2003;18:233-8. 
12. Yoo S, Teh E, Blinder R, Jolesz F. Modulation of cerebellar activities by acupuncture stimulation: evidence from fMRI study. Neuroimage 2004;22:932-40.

13. Yan B, Li K, Xu J, Wang W, Li K, Liu H, et al. Acupoint-specific fMRI patterns in human brain. Neurosci Lett 2005;383:236-40.

14. Treede R-D, Kenshalo DR, Gracely RH, Jones AKP. The cortical representation of pain. Pain 1999;79:105-11.

15. Derbyshire SWG, Jones AKP, Gyulai F, Clark S, Townsend D, Firestone LL. Pain processing during three levels of noxious stimulation produces differential patterns of central activity. Pain 1997;73:431-45.

16. Tracey I, Ploghaus A, Gati JS, Clare S, Smith S, Menon RS, et al. Imaging attentional modulation of pain in the periaqueductal gray in humans. J Neurosci 2002;22:2748-52.

17. Petrovic P, Kalso E, Petersson KM, Ingvar M. Placebo and opioid analgesia-imaging a shared neuronal network. Science 2002;295: $1737-40$.

18. Wager TD, Rilling JK, Smith EE, Sokolik A, Casey KL, Davidson RJ, et al. Placebo-induced changes in fMRI in the anticipation and experience of pain. Science 2004;303:1162-7.

19. Fuente-Fernandez R de la, Phillips AG, Zamburlini M, Sossi V, Calne DB, Ruth TJ, et al. Dopamine release in human ventral striatum and expectation of reward. Behav Brain Res 2002;136:359-63.

20. Chiu J-H, Chung M-S, Cheng H-C, Yeh T-C, Hsieh J-C, Chang C-Y, et al. Different central manifestations in response to electroacupuncture at analgesic and nonanalgesic acupoints in rats: a manganese-enhanced functional magnetic resonance imaging study. Can J Vet Res 2003;67: 94-101.

21. Liu W-C, Feldman SC, Cook DB, Hung D-L, Xu T, Kalnin AJ, et al. fMRI study of acupuncture-induced periaqueductal gray activity in humans. Neuroreport 2004;15:1937-40.

22. Wu MT, Sheen JM, Chuang KH, Yang P, Chin SL, Tsai CY, et al. Neuronal specificity of acupuncture response: a fMRI study with electroacupuncture. Neuroimage. 2002;16:1028-37.

23. Zhang WT, Jin Z, Cui GH, Zhang KL, Zhang L, Zeng YW, et al. Relations between brain network activation and analgesic effect induced by low vs. high frequency electrical acupoint stimulation in different subjects: a functional magnetic resonance imaging study. Brain Res 2003;982: $168-78$.
24. Wu MT, Hsieh JC, Xiong J, Yang CF, Pan HB, Chen YC, et al. Central nervous pathway for acupuncture stimulation: localization of processing with functional MR imaging of the brain-preliminary experience. Radiology 1999;212:133-41.

25. Hui KKS, Liu J, Makris N, Gollub RL, Chen AJW, Moore CI, et al. Acupuncture modulates the limbic system and subcortical gray structures of the human brain: evidence from fMRI studies in normal subjects. Hum Brain Mapp 2000;9:13-25.

26. Kong J, Ma L, Gollub R, Wei J, Yang X, Li D, et al. A pilot study of functional magnetic resonance imaging of the brain during manual and electroacupuncture stimulation of acupuncture point (LI-4 Hegu) in normal subjects reveals differential brain activation between methods. J Altern Complement Med 2002;8:411-9.

27. Hsieh J, Tu C, Chen F, Chen M, Yeh T, Cheng H, et al. Activation of the hypothalamus characterizes the acupuncture stimulation at the analgesic point in human: a positron emission tomography study. Neurosci Lett 2001;307:105-8

28. Fang JL, Krings T, Weidemann J, Meister IG, Thron A. Functional MRI in healthy subjects during acupuncture: different effects of needle rotation in real and false acupoints. Neuroradiology 2004;46:359-62.

29. Biella G, Sotgiu ML, Pellegata G, Paulesu E, Castiglioni I, Fazio F. Acupuncture produces central activations in pain regions. Neuroimage 2001;14:60-66.

30. Pariente J, White P, Frackowiak RSJ, Lewith G. Expectancy and belief modulate the neuronal substrates of pain treated by acupuncture. Neuroimage 2005;25:1161-67.

31. Berman BM, Lao L, Langenberg P, Lee WL, Gilpin AMK, Hochberg MC. Effectiveness of acupuncture as adjunctive therapy in osteoarthritis of the knee. Ann Intern Med 2004;141:901-10.

32. White P, Lewith GT, Prescott P, Conway J. Acupuncture versus placebo for the treatment of chronic mechanical neck pain. A randomised, controlled trial. Ann Intern Med 2004;141:911-20.

33. Lewith GT, Vincent $C$. The evaluation of the clinical effects of acupuncture. A problem reassessed and a framework for future research. Pain Forum 1995;4:29-39.

Received July 13, 2005; accepted July 20, 2005 


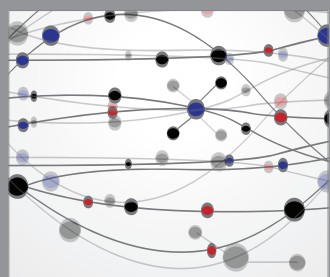

The Scientific World Journal
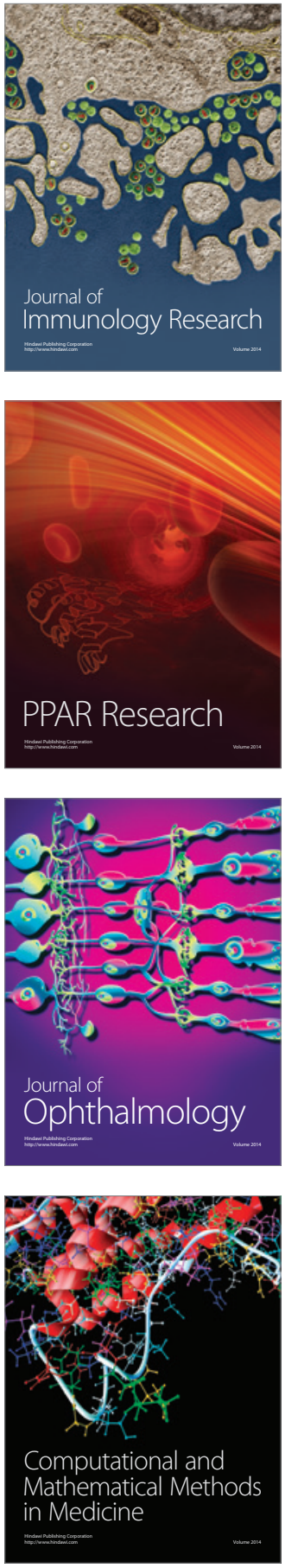

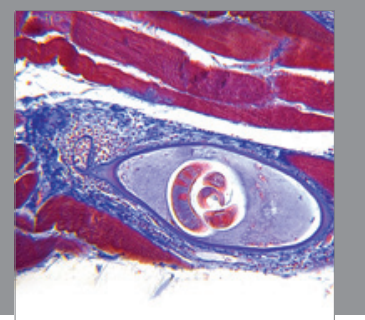

Gastroenterology

Research and Practice
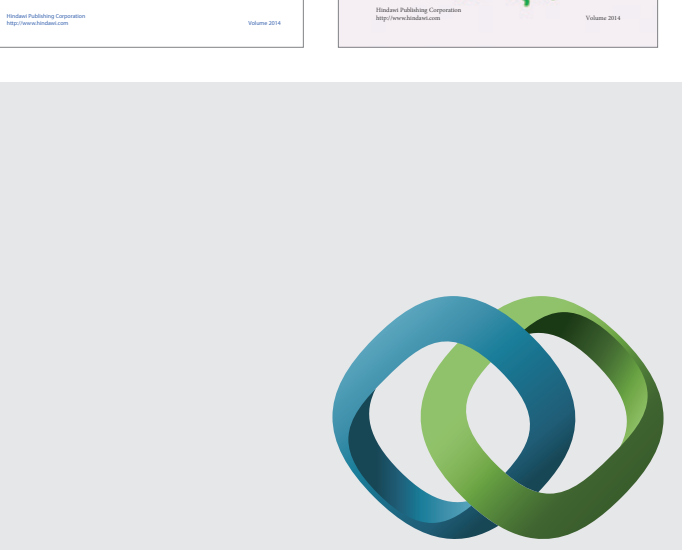

\section{Hindawi}

Submit your manuscripts at

http://www.hindawi.com
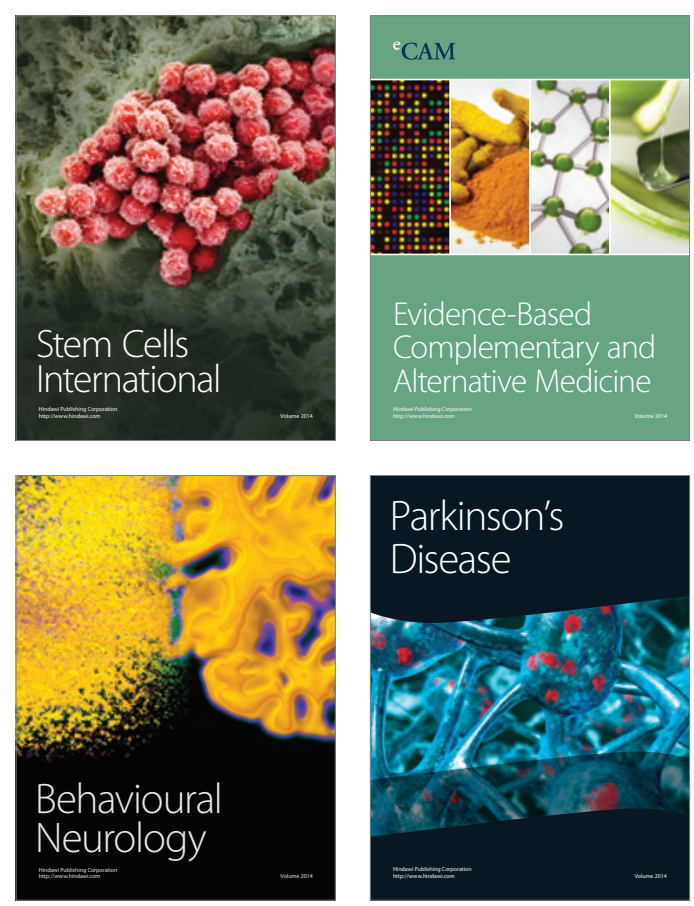

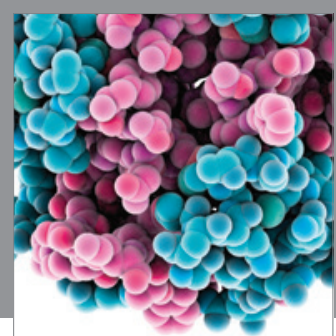

Journal of
Diabetes Research

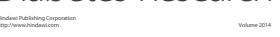

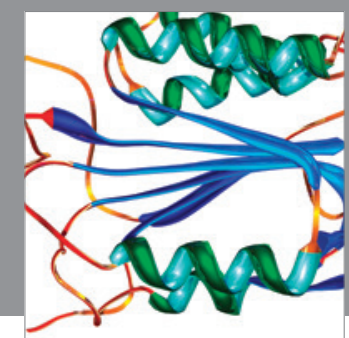

Disease Markers
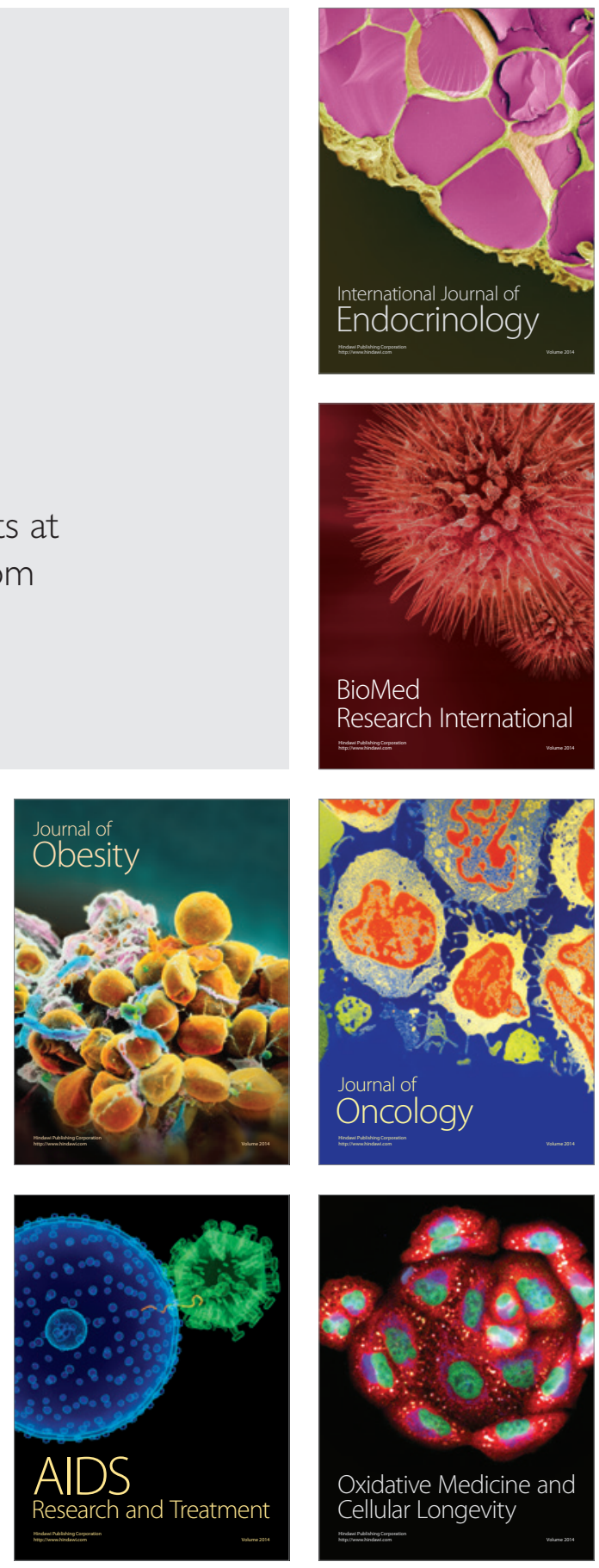